\title{
QUANTUM OSTROWSKI INEQUALITIES FOR q-DIFFERENTIABLE CONVEX FUNCTIONS
}

\author{
Muhammad Aslam Noor, MuHammad Uzair Awan \\ AND KHALIDA INAYAT NOOR
}

\begin{abstract}
In this paper, we establish a new quantum analogue of classical integral identity. Using this quantum integral identity, we derive some quantum analogues of Ostrowski type inequalities for $q$-differentiable convex functions.
\end{abstract}

Mathematics subject classification (2010): 26D15, 26A51.

Keywords and phrases: Convex functions, quantum, $q$-differentiable, $q$-integration, Ostrowski.

\section{REFERENCES}

[1] P. Cerone, S. S. Dragomir, Ostrowski type inequalities for functions whose derivatives satisfy certain convexity assumptions, Demonstr. Math. 37 (2) 299-308, (2004).

[2] S. S. Dragomir, T. M. Rassias, Ostrowski Type Inequalities and Applications in Numerical Integration, Springer Netherlands, 2002.

[3] T. ERnst, A Comprehensive Treatment of q-Calculus, Springer Basel Heidelberg New York Dordrecht London, 2012.

[4] T. ERnst, A Method for q-Calculus, J. Nonl. Math. Phy. 10 (4) 487-525, (2003).

[5] H. Gauchman, Integral inequalities in q-calculus, Comput. Math. Appl. 47 281-300, (2004).

[6] M. A. Noor, M. U. Awan, K. I. Noor, Some quantum estimates for Hermite-Hadamard inequalities, Appl. Math. Comput. 251 675-679, (2015).

[7] M. A. Noor, K. I. Noor, M. U. AwAn, Some quantum integral inequalities via preinvex functions, Appl. Math. Comput., in press.

[8] M. A. NOor, K. I. Noor, M. U. AwAn, F. SAFdAR, Some quantum analogues of integral inequalities, Appl. Math. Comput., to appear.

[9] C. E. M. PEARCE, J. PECARIC, Inequalities for differentiable mappings with application to special means and quadrature formulae, Appl. Math. Lett. 13 51-55, (2000).

[10] W. SudsutAD, S. K. NTOUYAS, J. TARIBOON, Quantum integral inequalities for convex functions, J. Math. Inequal. 9 (3) (2015), 781-793.

[11] J. TARiBoon, S. K. Ntouyas, Quantum integral inequalities on finite intervals, J. Inequal. App. 2014, 121 (2014).

[12] J. TARIBOON, S. K. NTOUYAS, Quantum calculus on finite intervals and applications to impulsive difference equations, Adv. Differ. Equ. 2013, 282 (2013). 